\author{
Maja Fošner • Nadeem ur Rehman · Tarannum Bano
}

\title{
A note on generalized derivations on prime rings
}

Received: 21 April 2015 / Accepted: 29 November 2017 / Published online: 29 December 2017

(C) The Author(s) 2017. This article is an open access publication

\begin{abstract}
Let $R$ be a prime ring with the extended centroid $C$ and symmetric Martindale quotient ring $Q_{s}(R)$. In this paper we prove the following result. Let $F: R \rightarrow R$ be a generalized derivation associated with a non-zero derivation $d$ on $R$ and let $h$ be an additive map of $R$ such that $F(x) x=x h(x)$ for all $x \in R$. Then either $R$ is commutative or $F(x)=x p$ and $h(x)=p x$ where $p \in Q_{s}(R)$.
\end{abstract}

\section{Mathematics Subject Classification 16 N60 $\cdot 16$ W25}

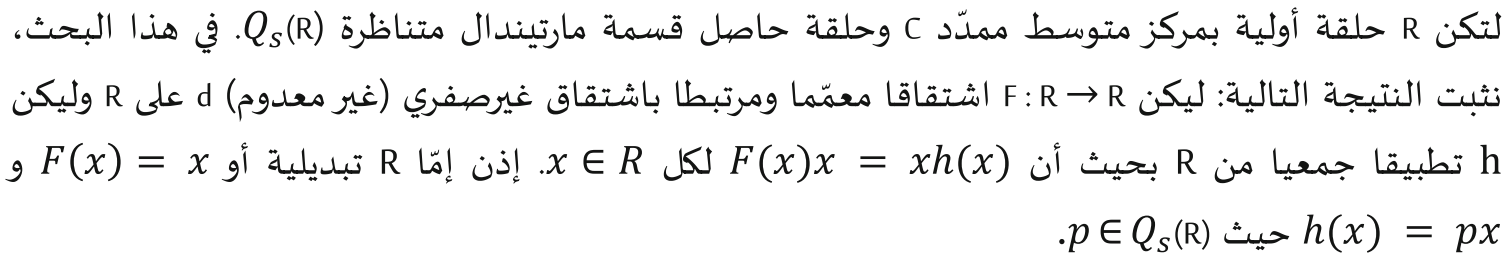

\section{Introduction}

Throughout the paper, $R$ will be an associative ring with center $Z$. Recall that $R$ is prime if for any $a, b \in R$, $a R b=0$ implies that $a=0$ or $b=0$. By $Q_{l}(R)$ and $Q_{r}(R)$ we denote the left Martindale ring of quotients of $R$ and the right Martindale ring of quotients of $R$, respectively. Further, we denote by $Q_{s}(R)$ the symmetric Martindale quotients ring of ring $R$. The center $C$ of $Q_{s}(R)$ is a field and it is the center of both $Q_{l}(R)$ and $Q_{r}(R)$. It is called the extended centroid of $R$. Also it is easily seen that $C$ is the centralizer of $R$ in both $Q_{r}(R)$ and $Q_{l}(R)$. In particular, $Z \subseteq C$. The subring of $Q_{r}(R)$ (or $Q_{l}(R)$ ) generated by $R$ and $C$ is called the central closure of $R$ and will be denoted by $R_{C}$. Another subring of $Q_{r}(R)$ is $Q_{s}(R)=\left\{q \in Q_{r} \mid I q \subseteq R\right.$

\section{Fošner (凶)}

Faculty of Logistics, University of Maribor, Mariborska cesta 7, 3000 Celje, Slovenia

E-mail: maja.fosner@um.si

N. ur Rehman · T. Bano

Department of Mathematics, Aligarh Muslim University, Aligarh 202002, India

E-mail: rehman100@gmail.com

T. Bano

E-mail: tarannumdlw@gmail.com 
for some nonzero ideal $I$ of $R$ \}. It is called the symmetric Martindale ring of quotients. We point out that $R \subseteq R_{C} \subseteq Q_{s}(R) \subseteq Q_{r}(R)$. Note that $q_{1} R q_{2}=0$, where $q_{1}, q_{2} \in Q_{l}(R)$ or $q_{1}, q_{2} \in Q_{r}(R)$ implies that $q_{1}=0$ or $q_{2}=0$. In particular, this shows that all $R_{C}, Q_{s}(R), Q_{l}(R)$, and $Q_{r}(R)$ are prime rings, so that one can construct (left, right, symmetric) Martindale ring of quotients and the central closure of each of these rings.

Let $R_{C} *_{C} C\{X\}$ be the free product over $C$ of $R_{C}$ and the free algebra over $C$ on an infinite set $X$ of indeterminates. A typical element in $R_{C} *_{C} C\{X\}$ is a sum of monomials of the form $\lambda a_{i_{0}} x_{j_{1}} a_{i_{1}} x_{j_{2}} \ldots x_{j_{n}} a_{i_{n}}$ where $\lambda \in C, a_{i_{k}} \in R_{C}$ and $x_{j_{k}} \in X . R$ satisfy a generalized polynomial identity over $C$ (simply $R$ is a GPI ring) if there exists a nonzero polynomial $p\left(x_{1}, x_{2}, \ldots, x_{n}\right) \in R_{C} *_{C} C\{X\}$ such that $p\left(r_{1}, r_{2}, \ldots, r_{n}\right)=0$ for all $r_{1}, r_{2}, \ldots, r_{n} \in R$. We refer the reader to [2,3] for more details.

An additive map $d: R \rightarrow R$ is called a derivation if $d(x y)=d(x) y+x d(y)$ holds for all $x, y \in R$. In particular, for a fixed $a \in R$, the map $I_{a}: R \rightarrow R$ given by $I_{a}(x)=[x, a]$ is a derivation called an inner derivation. Let $S$ be a non-empty subset of $R$. A map $f: R \rightarrow R$ is said to be centralizing on $S$ if $[f(x), x] \in Z$ for every $x \in S$. In special case where $[f(x), x]=0$ for every $x \in S$, the map $f$ is called commuting on $S$. The study of centralizing maps was initiated by a well-known theorem of Posner [15] which states that the existence of a nonzero centralizing derivation on a prime ring $R$ implies that $R$ is commutative. A number of authors have extended Posner's theorem in several ways. They have showed that nonzero derivations cannot be centralizing on various subsets of noncommutative prime rings (see [12] for probably the most general results of the kind), and similar conclusion hold for some other maps. In [5] Brešar studied maps that are centralizing and additive, and no further assumption was required. The main result of [5] characterizes commuting additive maps on prime rings $R$ : every such map is of the form $x \mapsto \lambda x+h(x)$ where $\lambda \in C$, and $h$ is an additive map of $R$ into $C$. Later, Lanski [13] dealt with the situation where a nonzero derivation $d$ of a prime ring $R$ satisfies $c_{1} x d(y)+c_{2} d(x) y+c_{3} y d(x)+c_{4} d(y) x \in C$ for some $c_{i} \in C$ and all $x, y \in S$, where $S$ is a subset of $R$. Neglecting rings of characteristic 2, the conclusion was: either all $c_{i}=0$ or $R$ satisfies $S_{4}$, the standard identity of degree 4 (however, the exact statements are much more precise). The condition considered by Lanski clearly covers the case of centralizing derivations, namely a linearization of $[d(x), x] \in Z$ gives $x d(y)-d(x) y+y d(x)-d(y) x \in Z$. The same is true for the case skew-centralizing on $S$ if $f(x) x+x f(x) \in Z$ for all $x \in S$. In the special case where $f(x) x+x f(x)=0$ for all $x \in S$, the map $f$ is called skew-commuting on $S$. In [6] Brešar proved that there is no nonzero additive maps that are skew-commuting on ideals of prime rings of characteristic not 2 .

An additive map $F: R \rightarrow R$ is called a generalized inner derivation if $F(x)=a x+x b$ for fixed $a, b \in R$. For such a map $F$, it is easy to see that $F(x y)=F(x) y+x[y, b]=F(x) y+x I_{b}(y)$ for all $x, y \in R$. This observation leads to the following definition, given in [4] and [9]; an additive map $F: R \rightarrow R$ is called a generalized derivation with associated derivation $d$ if $F(x y)=F(x) y+x d(y)$ holds for all $x, y \in R$.

Familiar examples of generalized derivations are derivations and generalized inner derivations and the later includes left multiplier, i.e., an additive map $F: R \longrightarrow R$ satisfying $F(x y)=F(x) y$ for all $x, y \in R$. In [11], Lee extended the definition of a generalized derivation as follows: by a generalized derivation we mean an additive mapping $F: I \rightarrow U$ such that $F(x y)=F(x) y+x d(y)$ holds for all $x, y \in I$, where $I$ is a dense left ideal of $R, U$ is the Utumi quotient ring (i.e., the maximal right quotient ring) of $R$ and $d$ is a derivation from $I$ to $U$. Moreover, Lee also proved that every generalized derivation can be uniquely extended to a generalized derivation of $U$, and thus all generalized derivations of $R$ will implicitly assumed to be defined on the whole of $U$. Lee obtained the following: every generalized derivation $F$ on a dense left ideal of $R$ can be extended to $U$ and assumes the form $F(x)=a x+d(x)$ for some $a \in U$ and a derivation $d$ on $U$.

Motivated by the work of Brešar [5] and Lanski [13], in this paper we consider $F$ as a generalized derivation and $h$ as an additive map of $R$ such that $F(x) x=x h(x)$ for all $x \in R$. In fact, our result extends Posner's Theorem [15], Brešar's Theorem [5] and Ashraf et al. [1].

\section{Main result}

In this section we shall prove our main theorem. Before that we need some known results:

Lemma 2.1 [14, Theorem 3] A prime ring $R$ satisfies a GPI if and only if $R_{C}$ is a primitive ring with nonzero socle and $e R_{C} e$ is a finite-dimensional division algebra over $C$ for each primitive idempotent e in $R_{C}$.

Lemma 2.2 [7, Main Theorem] Let $R$ be prime ring and let $n, m, k, l$ be positive integers. Suppose that

$$
\Sigma_{i=1}^{n} F_{i}(y) x a_{i}+\Sigma_{i=1}^{m} G_{i}(x) y b_{i}+\Sigma_{i=1}^{k} c_{i} y H_{i}(x)+\Sigma_{i=1}^{l} d_{i} x K_{i}(y)=0
$$


for all $x, y \in R$, where $F_{i}, G_{i}, H_{i}, K_{i}: R \rightarrow R_{C}$ are additive maps and $\left\{a_{1}, \ldots, a_{n}\right\},\left\{b_{1}, \ldots, b_{m}\right\},\left\{c_{1}, \ldots\right.$, $\left.c_{k}\right\},\left\{d_{1}, \ldots, d_{l}\right\}$ are $C$-independent subsets of $R$. Then one of the two possibilities holds:

(i) $R_{C}$ is a primitive ring with nonzero socle and $e R_{C}$ e is a finite-dimensional division algebra over $C$ for each primitive idempotent e in $R_{C}$ ( that is, $R$ is a GPI ring),

(ii) There exists elements $q_{i j} \in Q_{s}\left(R_{C}\right), i=1, \ldots, l, j=1, \ldots, m, p_{i j} \in Q_{s}\left(R_{C}\right), i=1, \ldots, k, j=$ $1, \ldots, n$ and additive maps $\lambda_{i j}: R \rightarrow C, i=1, \ldots, l, j=1, \ldots, n, \mu_{i j}: R \rightarrow C, i=1, \ldots, m, j=$ $1, \ldots, k$, such that

$$
\begin{aligned}
& F_{i}(y)=\Sigma_{j=1}^{k} c_{j} y p_{j i}+\Sigma_{j=1}^{l} \lambda_{j i}(y) d_{j}, \text { for all } y \in R, i=1, \ldots, n, \\
& G_{i}(x)=\Sigma_{j=1}^{l} d_{j} x q_{j i}-\Sigma_{j=1}^{k} \mu_{i j}(x) c_{j}, \text { for all } x \in R, i=1, \ldots, m, \\
& H_{i}(x)=-\Sigma_{j=1}^{n} p_{i j} x a_{j}+\Sigma_{j=1}^{m} \mu_{j i}(x) b_{j}, \text { for all } x \in R, i=1, \ldots, k, \\
& K_{i}(y)=-\Sigma_{j=1}^{m} q_{i j} y b_{j}-\Sigma_{j=1}^{l} \lambda_{j i}(y) a_{j}, \text { for all } y \in R, i=1, \ldots, l .
\end{aligned}
$$

Lemma 2.3 [10, Theorem 2] Let $R$ be a prime ring, $U$ its maximal right quotient ring and $I_{R}$ a dense $R$-submodule of $U_{R}$. Then I and $U$ satisfy the same differential identities

Now we are well equipped to prove our theorem:

Theorem 2.4 Let $R$ be a prime ring and $F: R \rightarrow R$ be a generalized derivation associated with a non-zero derivation $d$. Further let $h$ be an additive map of $R$ such that $F(x) x=x h(x)$ for all $x \in R$. Then either $R$ is commutative or $F(x)=x p$ and $h(x)=p x$ where $p \in U$.

Proof We have $F(x) x=x h(x)$ for all $x \in R$. Linearizing this relation we have

$$
F(y) x+F(x) y-y h(x)-x h(y)=0
$$

for all $x, y \in R$. We solve this functional identity in two different cases.

Case I: $R$ is not a GPI ring. Using Lemma 2.2, we get from (1)

$$
\begin{aligned}
& F(y)=y p+\lambda(y), \\
& F(x)=x q+\mu(x), \\
& h(x)=p x+\mu(x), \\
& h(y)=q y+\lambda(y),
\end{aligned}
$$

where $\lambda, \mu: R \rightarrow C$ additive maps. From (2), we have $F(y)-y p=\lambda(y) \in C$. Let $G$ be the additive map defined as $G(y)=F(y)-y p$ for any element $y \in R$. Since $F$ is a generalized derivation with associated derivation $d$, first we prove that $G$ is a generalized derivation of $R$.

$$
\begin{aligned}
G(x y) & =F(x y)-x y p=F(x) y+x d(y)-x y p \\
& =F(x) y+x d(y)-x y p+(x p y-x p y) \\
& =(F(x)-x p) y+x(d(y)+[p, y]) \\
& =G(x) y+x g(y),
\end{aligned}
$$

where $g(x)=d(x)+[p, x]$ is the associated derivation of $G$. Hence $G$ is a generalized derivation. Thus, by (2) $G(y)$ is central in $R$, for any element $y \in R$. Hence by Hvala [9, Lemma 3] either $R$ is commutative or $G=0$, which imply $F(y)-y p=0$ and hence $F(y)=y p$ for any $y \in R$. Similarly from (3), we find that either $R$ is commutative or $F(x)=x q$. These two relations imply that $p=q$ and $\lambda=\mu=0$ and hence $h(x)=p x$ where $p \in U$.

Case II: $R$ is a GPI ring. If there exists a nonzero idempotent $e$ in $R_{C}$. If there exists $e^{2}=e \neq\{0,1\}$ in $Q_{s}(R)$. Therefore, we can find a nonzero ideal $I$ of $R$ satisfying $e I+I e \subseteq R$. Then from (1), we get

$$
F(e y) e x+F(e x) e y=e x h(e y)+e y h(e x), \text { for all } x, y \in I \text {. }
$$

Thus

$$
F(e y) e x+F(e x) e y=e\{F(e y) e x+F(e x) e y\}, \text { for all } x, y \in I \text {. }
$$


This implies that

$$
(1-e) F(e y) e x+(1-e) F(e x) e y=0, \text { for all } x, y \in I .
$$

By Lemma 2.3, $Q_{s}(R)$ and $I$ satisfy the same differential identity. Thus we have

$$
(1-e) F(e y) e x+(1-e) F(e x) e y=0 \text {, for all } x, y \in Q_{s}(R) .
$$

This can be written as

$$
H(x) y+H(y) x=0,
$$

where $H(x)=(1-e) F(e x) e$. Replacing $x$ by $x z$ in (6), we get

$$
H(x z) y+H(y) x z=0 .
$$

Multiplying (6) by $z$ from the right, we find that

$$
H(x) y z+H(y) x z=0 .
$$

Comparing (7) and (8), we find that

$$
H(x z) y=H(x) y z .
$$

Replacing $y$ by $y u$ in (9), we get

$$
H(x z) y u=H(x) y u z .
$$

Right multiplication of (9) by $u$, we get

$$
H(x z) y u=H(x) y z u .
$$

Comparing (10) and (11), we get $H(x) y[z, u]=0$ for all $x, y, z, u \in Q_{s}(R)$. Since $R$ is prime and $Q_{s}(R)$ is also prime, we get from the last relation either $R$ is commutative or $H(x)=0$ for all $x \in Q_{s}(R)$. If $H(x)=0$, we get $(1-e) F(e x) e=0$ and considering $F(x)=a x+d(x)$ for $a \in U$, we find that

$$
(1-e)[a e x+d(e x)] e=0 .
$$

This implies

$$
(1-e) a e x e+(1-e) d(e) x e=0 .
$$

Since $R$ is prime and $e$ is non-trivial, we get $(1-e) a e+(1-e) d(e)=0$ for all non-trivial idempotents $e \in Q_{s}(R)$. Replacing $e$ by $1-e$ we get $e a(1-e)+e d(-e)=0$. Combining these two relations we get $d(e)+[a, e]=0$. Let $E$ be an additive subgroup generated by idempotents in $Q_{s}(R)$. Therefore, $d(u)+[a, u]=$ 0 for all $u \in E$. Now, for all $u, v \in E$, we get $d(u v)+[a, u v]=0=(d(u)+[a, u]) v+u(d(v)+[a, v])$. That is, $d(u)+[a, u]=0$ for all $u \in \bar{E}=[E, E]$. Now $[E, E] \neq 0$, since $[e, e+e x(1-e)] \neq 0$ for some $x \in Q_{s}(R)$. By Herstein's arguments $[8$, page 4$] 0 \neq Q_{s}[E, E] Q_{s} \subseteq \bar{E}, W=Q_{s}[E, E] Q_{s}$ is a nonzero ideal of $Q_{s}$. Therefore, $d(u)+[a, u]=0$ for all $u \in W$ and hence $d(x)+[a, x]=0$ for all $x \in Q_{s}(R)$ by Lemma 2.3. Thus $F(x)=a x+d(x)=a x-[a, x]=x a$.

By Martindale's theorem [14, Theorem 3] we know that $R_{C}$ is a primitive ring and $H=\operatorname{soc}\left(R_{C}\right) \neq 0$ and $e R_{C} e$ is a finite dimensional for any minimal idempotent $e$. If $H$ contains no non-trivial idempotent, then $H$ is a finite-dimensional division algebra over $C$. If soc $\left(R_{C}\right)$ contains no nontrivial idempotent, then soc $\left(R_{C}\right)$ must be a finite-dimensional division algebra over $C$, by [14, Theorem 3]. Since soc $\left(R_{C}\right)$ is a nonzero ideal of $R_{C}$, it follows $R_{C}=\operatorname{soc}\left(R_{C}\right)$ is a division algebra. Then for $x \neq 0 \in R$ we have from the given condition $h(x)=x^{-1} F(x) x$ in $R_{C}$. For any $x, y \neq 0 \in R$ we get from (1)

$$
F(x) y+F(y) x=x y^{-1} F(y) y+y x^{-1} F(x) x .
$$

Let $x y^{-1}=u$ in (12), we get

$$
F(u y) y+F(y) u y=u F(y) y+u^{-1} F(u y) u y \text { for all } u, y(\neq 0) \in R,
$$


and this implies that

$$
u F(u y) y+u F(y) u y=u^{2} F(y) y+F(u y) u y \text { for all } 0 \neq u, y \in R .
$$

This can be written as

$$
u F(u y)+u F(y) u=u^{2} F(y)+F(u y) u \text { for all } u, y \in R .
$$

Since $F(x)=a x+d(x)$ for $a \in Q_{s}(R)$, we get from the last relation

$$
u a u y+u d(u y)+u a y u+u d(y) u=u^{2} a y+u^{2} d(y)+a u y u+d(u y) u .
$$

The above relation for $y=1$ gives us $u a u+u d(u)+u a u=u^{2} a+a u^{2}+d(u) u$. This implies that $[u, d(u)]=[u,[u, a]]$, hence $d(u)=[u, a]$; now we get $F(x)=a x+x a-a x=x a$ and by our assumption that $F(x) x=x h(x)$, we get $h(x)=a x$, which completes the proof.

Open Access This article is distributed under the terms of the Creative Commons Attribution 4.0 International License (http:// creativecommons.org/licenses/by/4.0/), which permits unrestricted use, distribution, and reproduction in any medium, provided you give appropriate credit to the original author(s) and the source, provide a link to the Creative Commons license, and indicate if changes were made.

\section{References}

1. Ashraf, M.; Rehman, N.; Ali, S.; Mozumder, M.R.: On semiprime rings with generalized derivations. Bol. Soc. Parana de Mat. 28, 15-22 (2010)

2. Beidar, K.I.; Brešar, M.; Chebotar, M.A.: Functional identities revisited: the functional and the strong degree. Commun. Algebra 30, 935-969 (2002)

3. Beidar, K.I.; Martindale III, W.S.; Mikhalev, A.V.: Rings with Generalized Identities, Monographs and Textbooks in Pure and Applied Mathematics, vol. 196. Marcel Dekker, Inc., New York (1996)

4. Brešar, M.: On the distance of the composition of two derivations to the generalized derivations. Glasgow Math. J. 33, 89-93 (1991)

5. Brešar, M.: Centralizing mappings and derivations in prime rings. J. Algebra 156, 385-394 (1993)

6. Brešar, M.: On skew-commuting mappings of rings. Bull. Aust. Math. Soc. 47, 291-296 (1993)

7. Brešar, M.: Functional identities of degree two. J. Algebra 172, 690-720 (1995)

8. Herstein, I.N.: Topics in Ring Theory. University of Chicago Press, Chicago (1969)

9. Hvala, B.: Generalized derivations in rings. Commun. Algebra 26, 1147-1166 (1998)

10. Lee, T.K.: Semiprime rings with differential identities. Bull. Inst. Math. Acad. Sinica 20(1), 27-38 (1992)

11. Lee, T.K.: Generalized derivations of left faithful rings. Commun. Algebra 27(8), 4057-4073 (1999)

12. Lanski, C.: Differental identities, Lie ideals, and Posner's theorems. Pac. J. Math. 134, 275-297 (1988)

13. Lanski, C.: Lie ideals and central identities with derivation. Can. J. Math. 44, 553-560 (1992)

14. Martindale III, W.S.: Prime rings satisfying a generalized polynomial identity. J. Algebra 12, 576-584 (1969)

15. Posner, E.C.: Derivations in prime rings. Proc. Am. Math. Soc. 8, 1093-1100 (1957)

Publisher's Note Springer Nature remains neutral with regard to jurisdictional claims in published maps and institutional affiliations. 